\title{
Overview of the GRAMS (Gamma-Ray and AntiMatter Survey) Project
}

\section{Tsuguo Aramaki ${ }^{a, *}$ on behalf of the GRAMS Collaboration}

(a complete list of authors can be found at the end of the proceedings)

${ }^{a}$ Northeastern University, Department of Physics

360 Huntington Avenue, Boston, MA 02115, USA

E-mail: t.aramaki@northeastern.edu

GRAMS (Gamma-Ray and AntiMatter Survey) is a next-generation proposed balloon/satellite mission that will be the first to target both $\mathrm{MeV}$ gamma-ray observations and antimatter-based indirect dark matter searches with a LArTPC (Liquid Argon Time Projection Chamber) detector. Astrophysical observations at $\mathrm{MeV}$ energies have been poorly explored and long-neglected. With a cost-effective, large-scale LArTPC, a single LDB (Long-Duration Balloon) flight could provide an order of magnitude improved sensitivity compared to previous experiments. We can uniquely measure gamma rays from annihilating dark matter and evaporating primordial black holes. Additionally, GRAMS can extensively explore dark matter parameter space via antimatter measurements. In particular, low-energy antideuterons can be background-free dark matter signatures. We could deeply investigate the parameter space and validate the potential dark matter signatures suggested by the Fermi gamma-ray observations and AMS-02 antiparticle measurements.

$37^{\text {th }}$ International Cosmic Ray Conference (ICRC 2021)

July 12th-23rd, 2021

Online - Berlin, Germany

\footnotetext{
*Presenter
} 


\section{Introductions}

Astrophysical observations of gravitational lensing, the Bullet Cluster, and galaxy rotation curves have indicated the existence of dark matter since the 1960s [1, 2]. However, the nature and origin of dark matter are still unknown, and many theories and experiments have been proposed and are ongoing to reveal the mystery of dark matter. Direct dark matter search experiments, usually located at the underground lab, try to measure the recoil energy induced by the dark matter scattering inside the detector. On the other hand, indirect dark matter experiments measure Standard Model particles produced in dark matter annihilation/decay. The recent results of FermiLAT (Large Area Telescope on the Fermi Gamma-Ray Space Telescope) and AMS-02 (Alpha Magnetic Spectrometer) suggested possible dark matter signatures in gamma-ray and antiproton measurements, respectively [3-7]. However, these results are in strong tension with Fermi dwarf spheroidal galaxies observations, and a new project is needed to shed light on these issues.

Astrophysical observations via high-energy gamma-ray (above $20 \mathrm{MeV}$ ) and X-ray (up to 80 $\mathrm{keV}$ ) measurements have been well-explored by the Fermi-LAT (Large Area Telescope on the Fermi Gamma-Ray Space Telescope) and the NuSTAR (Nuclear Spectroscopic Telescope Array) missions, respectively $[8,9]$. Spectra of gamma-ray sources in the $\mathrm{MeV}$ energy range (MeV gamma rays), however, have not yet been well-explored, leading to the so-called "MeV gap." COMPTEL (The Imaging COMPton TELescope) produced the first catalog of MeV sources but only with approximately 30 objects [10]. The $\mathrm{MeV}$ regime can potentially provide rich information on astrophysical processes. Nuclear lines from the radioactive isotopes are mainly in the $\mathrm{MeV}$ range. They are the keys to understanding nucleosynthesis processes and mechanisms of astrophysical phenomena, including relativistic flows generated in stellar-mass black holes, supermassive black holes in active galactic nuclei, and various types of neutron stars such as radio pulsars and magnetars [11]. Moreover, $\mathrm{MeV}$ gamma rays could be produced in dark matter annihilation/decay and primordial black hole evaporation and association with gravitational waves from neutron-star mergers [12].

GRAMS (Gamma-Ray and AntiMatter Survey) is a proposed balloon-borne/satellite-based mission to target both astrophysical observations with $\mathrm{MeV}$ gamma rays and indirect dark matter searches with antimatter using a LArTPC (Liquid Argon Time Projection Chamber) detector. The LArTPC is cost-effective and easily expandable to build a large-scale detector, unlike current and previous experiments with semiconductors or scintillation crystals, allowing us to have extensive sensitivities to $\mathrm{MeV}$ gamma rays and antimatter. The performance of a LArTPC detector, in particular, has been significantly improved over the past decade for neutrino and dark matter search experiments [13].

\section{Detector Design}

The GRAMS instrument includes a LArTPC detector surrounded by two layers of plastic scintillators (see Figure 1). The overall instrument (outer layer of the plastic scintillators) will approximately be $3.5 \mathrm{~m} \times 3.5 \mathrm{~m} \times 2 \mathrm{~m}$, which is compatible with a payload for LDB (LongDuration Balloon) flights. The plastic scintillators can measure the time-of-flight (TOF) of an incoming antiparticle. They work as veto counters to reject incoming charged particles for gammaray measurements. The plastic scintillators will be operated at ambient temperature. The LArTPC 


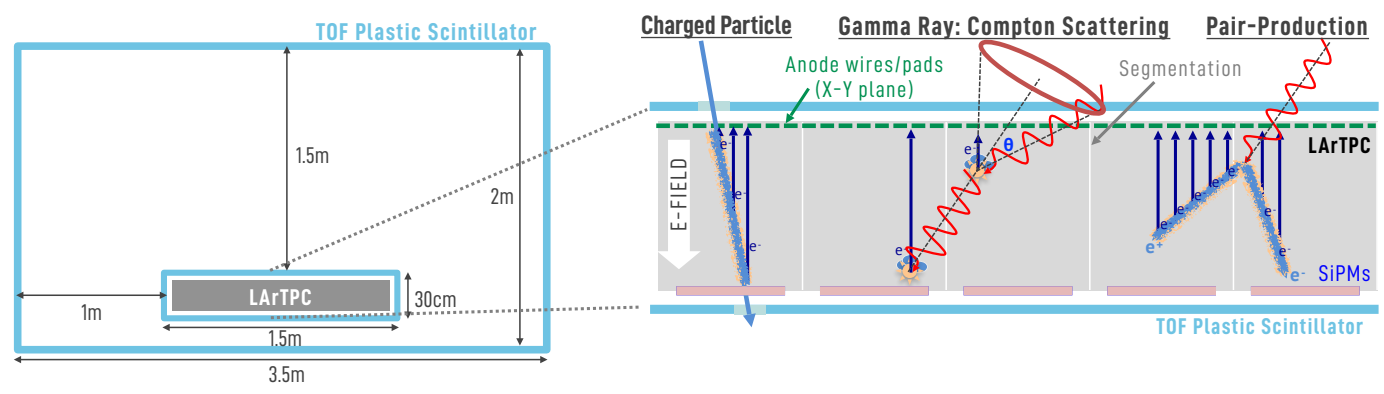

Figure 1: GRAMS detector design and detection concept for $\mathrm{MeV}$ gamma rays

will be about $1.4 \mathrm{~m} \times 1.4 \mathrm{~m} \times 0.2 \mathrm{~m}$ and will work as a calorimeter and particle tracker for antimatter detection while acting as a Compton camera for gamma-ray measurements.

The LArTPC is cost-effective since argon is one of the most abundant gases on earth. It will be operated at about 85 Kelvin in the liquid phase. We measure both scintillation light and ionization electrons emitted from excited and ionized argon atoms induced by the incoming particle. The ionization electrons will drift to the anode plane along the electric field applied inside the LArTPC. Silicon Photo-Multipliers (SiPMs) will measure the scintillation light, providing the trigger and timing of the event. The signals induced on the anode sensors wires or pads with a $\sim 2 \mathrm{~mm}$ pitch provide $x / y$ positions of the event. The satellite mission will have an upgraded design with a finer pitch of wires/pads. The drift time of the ionization electrons measured relative to the event-triggered time in SiPMs can provide the $z$ coordinate. Unlike semiconductors or scintillation detectors, the LArTPC does not need to configure a multi-layer design with readout electronics at each layer to reconstruct three-dimensional space points of the event. As a result, the LArTPC can significantly reduce the number of readout electronics and the total power consumption compared to other detectors for the same size, allowing to configure a large-scale design. There is almost no dead volume inside the LArTPC, which allows having a profound detection efficiency, unlike semiconductors or scintillation detectors with mounting frames and preamps nearby. The LArTPC can also have the capability to identify electron recoil events from nuclear recoil events, separating and rejecting neutron background events, as has been well demonstrated in dark matte search experiments. Table 1 shows the comparison between LArTPC and semiconductors or scintillation detectors.

\section{MeV Gamma-Ray Observation}

\subsection{Detection Concept}

While high-energy gamma rays tend to produce electron-positron pairs, the Compton scattering process dominantly occurs when a MeV gamma-ray below $10 \mathrm{MeV}$ enters the LArTPC. An incident gamma ray may undergo multiple Compton scatterings before being photo-absorbed or even escape from the sensitive volume. It can fully deposit its original energy inside the LArTPC or partially if the Compton scattered photon escapes from the detector. We estimated the effective area for the GRAMS balloon (satellite) flight using a GEANT4 simulation (see the right panel of Figure 2 ) [14]. Here, for reliable event reconstruction, we selected events for up to three Compton scatterings with vertices separated more than 10 (2) $\mathrm{cm}$ from each other and for pair-production 
with leaving $2(0.4) \mathrm{cm}$ or longer tracks of the electron and positron inside the detector. We have recently developed event reconstruction techniques to analyze four or more Compton scattering events inside the LArTPC, which will allow us to have a further enhanced effective area.
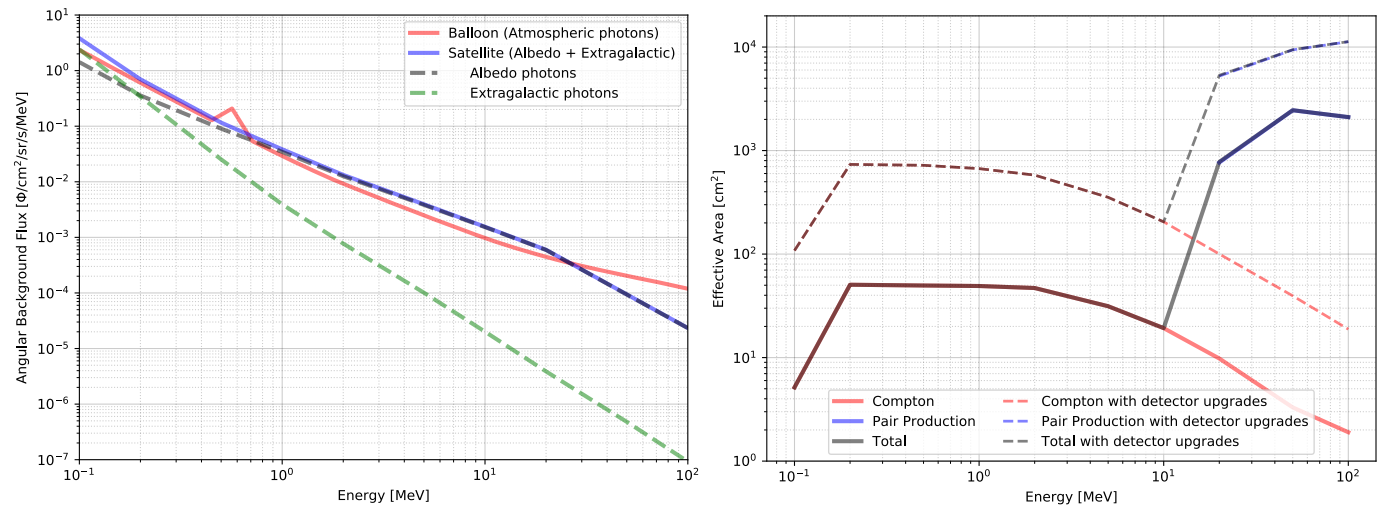

Figure 2: The left figure shows expected background fluxes for the balloon experiment (atmospheric photons) and the satellite mission in a low-earth orbit (Albedo and Extragalactic photons) [15]. The right figure shows the effective areas for Compton scattering and pair-production events in GRAMS (solid lines). The dashed lines represent the effective areas with detector upgrades.

\subsection{Sensitivity}

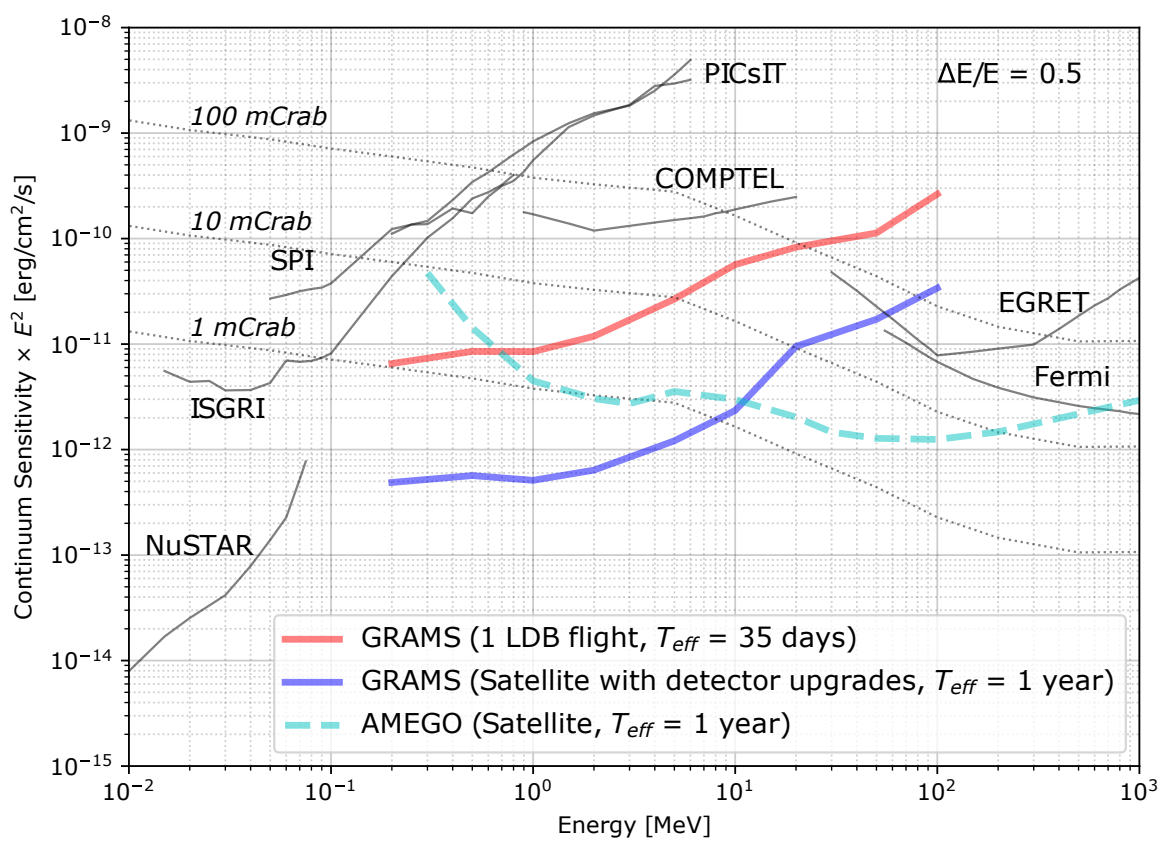

Figure 3: The continuum gamma-ray sensitivities at a $3 \sigma$ confidence level for the GRAMS balloon experiment (one LDB flight, $T_{\text {ef } f}=35$ days) and a possible satellite mission with detector upgrades $\left(T_{\text {ef } f}=1\right.$ year) compared to the sensitivities for previous and future experiments, including SPI (SPectrometer for INTEGRAL), PICsIT (PIxelated CsI Telescope) and ISGRI (INTEGRAL Soft Gamma-Ray Imager) on INTEGRAL. Black dashed lines represent the flux levels of 1-100 mCrab $[16,17]$.

We have estimated GRAMS sensitivities to gamma rays at a 3- $\sigma$ confidence level based on the effective area discussed above and the background model shown in the left panel of Figure 2. The 
atmospheric photons dominate the background for the balloon flight ${ }^{1}$, while the albedo photons dominate for the satellite mission in a low-earth orbit. The sensitivity for a single LDB flight with 35 days of observation time could be an order of magnitude improved compared to previous experiments [16]. We can expect further enhanced sensitivity for the GRAMS satellite mission, comparable to future proposed missions such as e-ASTROGAM and AMEGO [17]. GRAMS will also have further improved sensitivities to gamma-ray lines from positron annihilation and radioactive isotopes, as well as potential gamma-ray lines from Galactic neutron star merger remnants [18, 19].

\section{Antimatter Survey}

\subsection{Detection Concept}

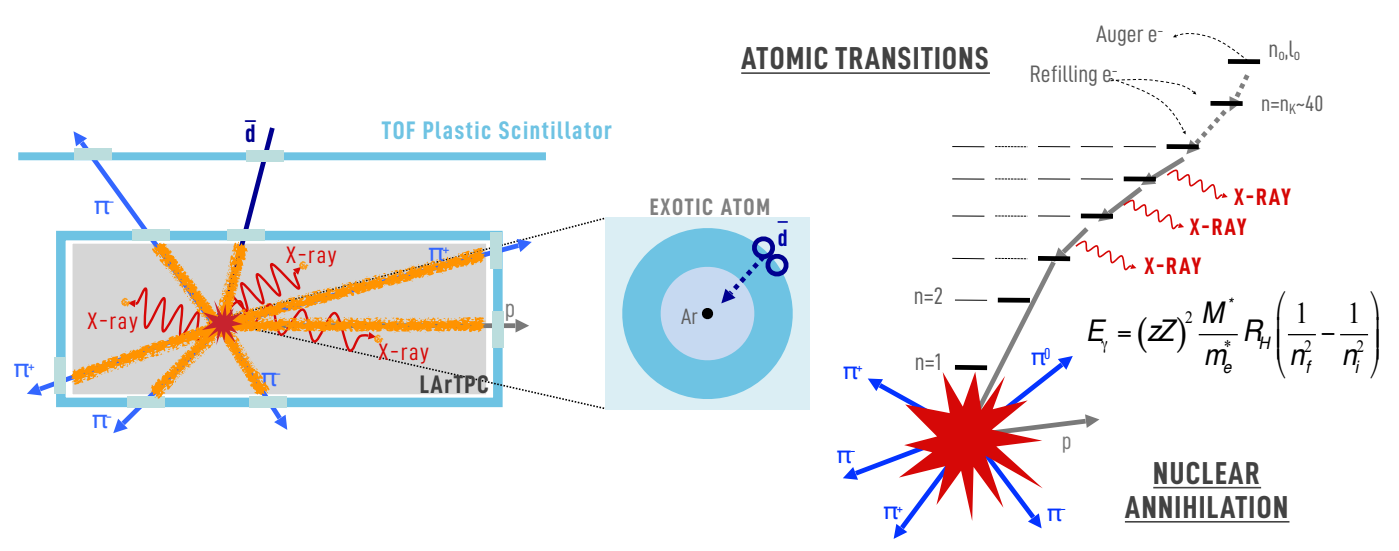

Figure 4: The GRAMS antimatter detection technique. The stopped antimatter forms an excited exotic atom that decays and emits atomic X-rays and annihilation products (pions and protons). The atomic X-rays, pion/proton multiplicities, and the stopping depth in the LArTPC provide the particle identification capability.

The GRAMS antimatter detection relies on capturing an antiparticle in a target material with the subsequent formation and decay of an exotic atom, similar to the GAPS project [20-22]. The antiparticle slows down and stops inside the LArTPC detector, where it forms an exotic atom in the excited state with an argon atom. Then, the exotic atom emits atomic X-rays as it de-excites, followed by the nuclear annihilation with the emission of pions and protons. Additionally, GRAMS measures the stopping range of the incoming antiparticles with an excellent position resolution in the LArTPC. Thus, the incoming antiparticle can be identified based on the atomic X-rays, the number of pions and protons, and the combination of the TOF, $\mathrm{dE} / \mathrm{dX}$ energy deposit, and the stopping range of the incoming particle. The detection concept and particle identification techniques were validated and demonstrated in the accelerator test with an antiproton beam as well as the prototype flight for the GAPS experiment [21, 23-25]. The expected mimic/background events could be as small as 0.01 during the LDB flight.

\subsection{Sensitivity}

The low-energy antideuteron measurements are considered essentially background-free dark matter searches since the signal-to-background ratio is more than two orders of magnitudes [26-28].

\footnotetext{
${ }^{1}$ The atmospheric photon flux was estimated using EXcel-based Program for calculating Atmospheric Cosmic ray Spectrum (EXPACS).
} 
The left panel of Figure 1 shows the predicted antideuteron fluxes from dark matter annihilation (primary) and cosmic ray interactions (secondary/background). The figure also includes the sensitivities for GRAMS and GAPS (three LDB flights, 105 days) and the current upper limit obtained by BESS [22, 29].

The recent results from the Fermi-LAT gamma-ray observations in the Galactic Center region and the AMS-02 antiproton measurements implied the detection of potential dark matter signatures [3-6, 30, 31]. The right panel of Figure 1 shows the areas in the dark matter parameter space that could explain these results. The figure also includes the GRAMS and GAPS sensitivities in the parameter space, taking into account the uncertainty of the antideuteron production model $[7,32,33]$. GAPS will start investigating these areas while GRAMS would be able to explore these regions extensively.
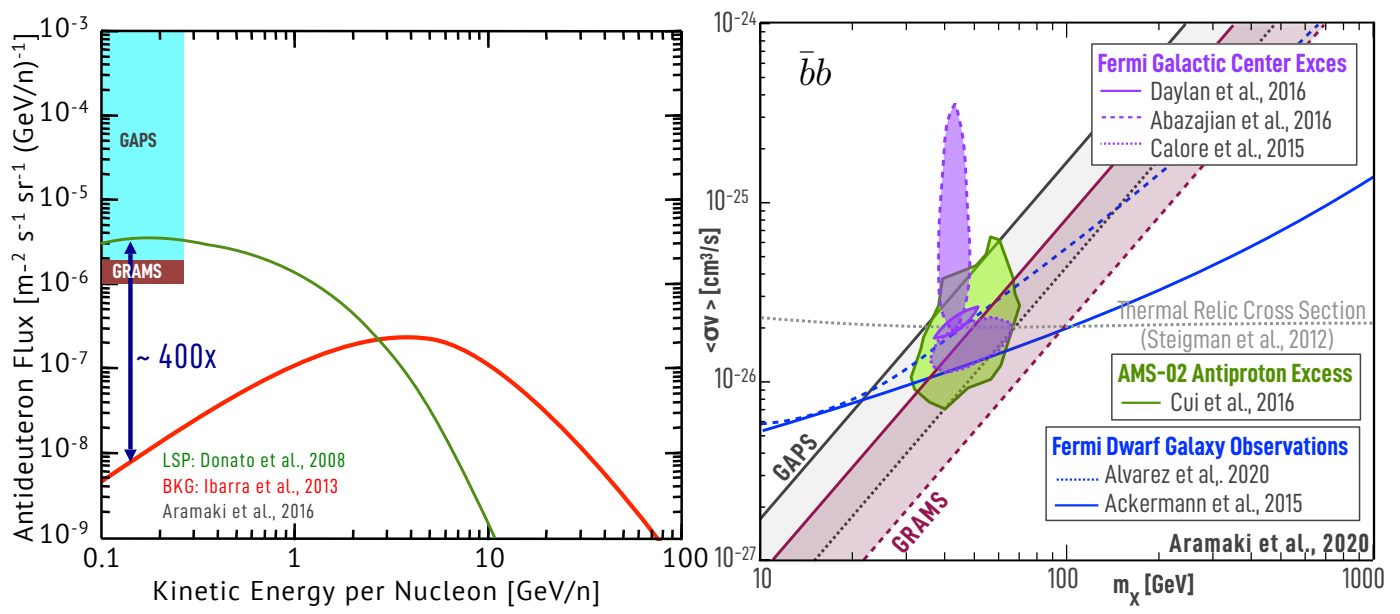

Figure 5: The left figure shows the antideuteron sensitivities for GRAMS (three LDB flights from Antarctica, 105 days) and other experiments together with the predicted antideuteron fluxes from dark matter annihilation (primary) and cosmic ray interactions (secondary) [22, 26-29, 34]. The right figure shows the parameter space for the possible dark matter signatures suggested by Fermi and AMS-02 [3-6, 30], where GRAMS and GAPS sensitivities are overlaid with an uncertainty of the antideuteron production model [7, 32, 33]. GAPS is an antideuteron search experiment using the exotic atom technique that is currently under construction for a science flight with a first opporunity in the 2022 austral summer.

\section{Conclusion and Outlook}

GRAMS is a proposed next-generation mission to target both gamma-ray observations in the poorly explored $\mathrm{MeV}$ energy band and indirect dark matter searches with antimatter. The project will begin with a balloon experiment as a step forward to a satellite mission. With a cost-effective, largescale LArTPC detector, the sensitivity to $\mathrm{MeV}$ gamma rays can be more than an order of magnitude improved compared to previous experiments with a single balloon flight. GRAMS antideuteron measurements can be essentially background-free dark matter searches. We can investigate and validate the possible dark matter detection indicated in the Fermi GCE and AMS-02 antiproton excess. The project is currently in the R\&D phase, and we have developed the event reconstruction techniques for multiple Compton scatterings. We are currently building a small-scale prototype detector, MiniGRAMS, to demonstrate the detection concept and the detector performance. 


\section{Acknowledgments}

This work was supported by Tsuguo Aramaki's start-up funds from Northeastern University. We acknowledge support from JSPS KAKENHI 610 grant numbers 20K22355 and 20H00153. We also acknowledge support from Barnard College and Columbia University.

\section{References}

[1] F. Zwicky, On the masses of nebulae and of clusters of nebulae, The Astrophysical Journal 86 (1937) 217.

[2] D. Clowe, A. Gonzalez, M. Markevitch, Weak-lensing mass reconstruction of the interacting cluster 1e 0657-558: Direct evidence for the existence of dark matter, The Astrophysical Journal 604 (2004) 596.

[3] F. Calore, I. Cholis, C. McCabe, C. Weniger, A tale of tails: dark matter interpretations of the fermi gev excess in light of background model systematics, Physical Review D 91 (2015) 063003.

[4] T. Daylan, D. P. Finkbeiner, D. Hooper, T. Linden, S. K. Portillo, N. L. Rodd, T. R. Slatyer, The characterization of the gamma-ray signal from the central milky way: A case for annihilating dark matter, Physics of the Dark Universe 12 (2016) $1-23$.

[5] K. N. Abazajian, R. E. Keeley, Bright gamma-ray galactic center excess and dark dwarfs: Strong tension for dark matter annihilation despite milky way halo profile and diffuse emission uncertainties, Physical Review D 93 (2016) 083514.

[6] M.-Y. Cui, Q. Yuan, Y.-L. S. Tsai, Y.-Z. Fan, A possible dark matter annihilation signal in the ams-02 antiproton data, arXiv preprint arXiv:1610.03840 (2016).

[7] M. Korsmeier, F. Donato, N. Fornengo, Prospects to verify a possible dark matter hint in cosmic antiprotons with antideuterons and antihelium, Physical Review D 97 (2018) 103011.

[8] W. Atwood, A. A. Abdo, M. Ackermann, W. Althouse, B. Anderson, M. Axelsson, L. Baldini, J. Ballet, D. Band, G. Barbiellini, et al., The large area telescope on the fermi gamma-ray space telescope mission, The Astrophysical Journal 697 (2009) 1071.

[9] F. A. Harrison, W. W. Craig, F. E. Christensen, C. J. Hailey, W. W. Zhang, S. E. Boggs, D. Stern, W. R. Cook, K. Forster, P. Giommi, et al., The nuclear spectroscopic telescope array (nustar) high-energy x-ray mission, The Astrophysical Journal 770 (2013) 103.

[10] V. Schönfelder, K. Bennett, J. Blom, H. Bloemen, W. Collmar, A. Connors, R. Diehl, W. Hermsen, A. Iyudin, R. Kippen, et al., The first comptel source catalogue, Astronomy and Astrophysics Supplement Series 143 (2000) 145-179.

[11] M. S. Longair, High energy astrophysics, Cambridge university press, 2011.

[12] B. Abbott, R. Abbott, R. Adhikari, A. Ananyeva, S. Anderson, S. Appert, K. Arai, M. Araya, J. Barayoga, B. Barish, et al., Multi-messenger observations of a binary neutron star merger, Astrophysical Journal Letters 848 (2017) L12.

[13] V. Chepel, H. Araújo, Liquid noble gas detectors for low energy particle physics, Journal of Instrumentation 8 (2013) R04001.

[14] S. Agostinelli, J. Allison, K. a. Amako, J. Apostolakis, H. Araujo, P. Arce, M. Asai, D. Axen, S. Banerjee, G. . Barrand, et al., Geant4-a simulation toolkit, Nuclear instruments and methods in physics research section A: Accelerators, Spectrometers, Detectors and Associated Equipment 506 (2003) 250-303.

[15] P. Cumani, M. Hernanz, J. Kiener, V. Tatischeff, A. Zoglauer, Background for a gamma-ray satellite on a low-earth orbit, Experimental Astronomy (2019) 1-30.

[16] T. Takahashi, L. Stawarz, Y. Uchiyama, Multiwavelength astronomy and cta: X-rays, Astroparticle Physics 43 (2012) 142-154.

[17] A. De Angelis, V. Tatischeff, M. Tavani, U. Oberlack, I. Grenier, L. Hanlon, R. Walter, A. Argan, P. von Ballmoos, A. Bulgarelli, et al., The e-astrogam mission, Experimental Astronomy 44 (2017) 25-82.

[18] T. Aramaki, P. O. H. Adrian, G. Karagiorgi, H. Odaka, Dual mev gamma-ray and dark matter observatory-grams project, Astroparticle Physics 114 (2020) 107-114.

[19] M.-R. Wu, P. Banerjee, B. D. Metzger, G. Martínez-Pinedo, T. Aramaki, E. Burns, C. J. Hailey, J. Barnes, G. Karagiorgi, Finding the remnants of the milky way's last neutron star mergers, arXiv preprint arXiv:1905.03793 (2019). 
[20] K. Mori, C. J. Hailey, E. A. Baltz, W. W. Craig, M. Kamionkowski, W. T. Serber, P. Ullio, A novel antimatter detector based on X-ray deexcitation of exotic atoms, The Astrophysical Journal 566 (2002) 604.

[21] T. Aramaki, S. Chan, W. Craig, L. Fabris, F. Gahbauer, C. Hailey, J. Koglin, N. Madden, K. Mori, H. Yu, et al., A measurement of atomic x-ray yields in exotic atoms and implications for an antideuteron-based dark matter search, Astroparticle Physics 49 (2013) 52-62.

[22] T. Aramaki, C. Hailey, S. Boggs, P. von Doetinchem, H. Fuke, S. Mognet, R. Ong, K. Perez, J. Zweerink, Antideuteron sensitivity for the gaps experiment, Astroparticle Physics 74 (2016) 6-13.

[23] P. von Doetinchem, T. Aramaki, N. Bando, S. Boggs, H. Fuke, F. Gahbauer, C. Hailey, J. Koglin, S. Mognet, N. Madden, S. Okazaki, R. Ong, K. Perez, T. Yoshida, J. Zweerink, The flight of the GAPS prototype experiment, Astropart. Phys. 54 (2014) 93 - 109.

[24] S. Mognet, T. Aramaki, N. Bando, S. Boggs, P. von Doetinchem, H. Fuke, F. Gahbauer, C. Hailey, J. Koglin, N. Madden, K. Mori, S. Okazaki, R. Ong, K. Perez, G. Tajiri, T. Yoshida, J. Zweerink, The prototype GAPS (pGAPS) experiment, Nucl. Instrum. Methods Phys. Res. A 735 (2014) $24-38$.

[25] H. Fuke, R. A. Ong, T. Aramaki, N. Bando, S. E. Boggs, P. Doetinchem, F. Gahbauer, C. J. Hailey, J. Koglin, N. Madden, et al., The pgaps experiment: an engineering balloon flight of prototype gaps, Advances in Space Research 53 (2014) 1432-1437.

[26] F. Donato, N. Fornengo, P. Salati, Antideuterons as a signature of supersymmetric dark matter, Physical Review D $62(2000) 43003$.

[27] F. Donato, N. Fornengo, D. Maurin, Antideuteron fluxes from dark matter annihilation in diffusion models, Physical Review D 78 (2008) 043506.

[28] A. Ibarra, S. Wild, Determination of the cosmic antideuteron flux in a monte carlo approach, Physical Review D 88 (2013) 023014.

[29] H. Fuke, T. Maeno, K. Abe, S. Haino, Y. Makida, S. Matsuda, H. Matsumoto, J. Mitchell, A. Moiseev, J. Nishimura, et al., Search for cosmic-ray antideuterons, Physical review letters 95 (2005) 081101.

[30] M. Ackermann, M. Ajello, A. Albert, W. Atwood, L. Baldini, J. Ballet, G. Barbiellini, D. Bastieri, R. Bellazzini, E. Bissaldi, et al., The fermi galactic center gev excess and implications for dark matter, The Astrophysical Journal 840 (2017) 43.

[31] A. Alvarez, F. Calore, A. Genina, J. Read, P. D. Serpico, B. Zaldivar, Dark matter constraints from dwarf galaxies with data-driven j-factors, Journal of Cosmology and Astroparticle Physics 2020 (2020) 004.

[32] N. Fornengo, L. Maccione, A. Vittino, Dark matter searches with cosmic antideuterons: status and perspectives, Journal of Cosmology and Astroparticle Physics 2013 (2013) 031.

[33] S. Acharya, J. Adam, D. Adamová, J. Adolfsson, M. M. Aggarwal, G. A. Rinella, M. Agnello, N. Agrawal, Z. Ahammed, N. Ahmad, et al., Production of deuterons, tritons, he 3 nuclei, and their antinuclei in p p collisions at $\mathrm{s}=0.9,2.76$, and 7 tev, Physical Review C 97 (2018) 024615.

[34] R. Ong, T. Aramaki, R. Bird, M. Boezio, S. Boggs, R. Carr, W. Craig, P. Von Doetinchem, L. Fabris, F. Gahbauer, et al., The gaps experiment to search for dark matter using low-energy antimatter, POS PROCEEDINGS OF SCIENCE (2017) 1-8. 


\section{Full Authors List: GRAMS Collaboration}

K. Aoyama ${ }^{1}$, T. Aramaki ${ }^{2}$, J. Asaadi ${ }^{3}$, L. Fabris ${ }^{4}$, Y. Ichinohe ${ }^{5}$, Y. Inoue ${ }^{6,7,8}$, G. Karagiorgi ${ }^{9}$, D. Khamgulyan ${ }^{10}$, M. Kimura ${ }^{1}$, J. Leyva ${ }^{2}$, R. Mukherjee ${ }^{11}$, T. Nakasone ${ }^{1}$, H. Odaka ${ }^{7,12,13}$, K. Perez ${ }^{14}$, M. Sakurai ${ }^{1}$, W. Seligman ${ }^{9}$, S.

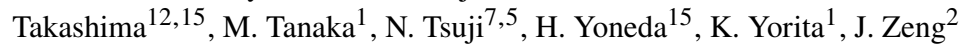

${ }^{1}$ Waseda University, 3-4-1, Okubo, Shinjuku, Tokyo 169-8555, Japan. ${ }^{2}$ Northeastern University, 360 Huntington Ave, Boston, MA 02115, USA. ${ }^{3}$ University of Texas-Arlington, Arlington, TX 76019, USA. ${ }^{4}$ Oak Ridge National Laboratory, Oak Ridge, TN 37830, USA. ${ }^{5}$ Department of Physics, Rikkyo University, 3-34-1 Nishi Ikebukuro, Toshima, Tokyo 171-8501, Japan. ${ }^{6}$ Department of Earth and Space Science, Graduate School of Science, Osaka University, Toyonaka, Osaka 560-0043, Japan. ${ }^{7}$ Interdisciplinary Theoretical \& Mathematical Science Program (iTHEMS), RIKEN, 2-1 Hirosawa, Wako, Saitama 351-0198, Japan. ${ }^{8}$ Kavli Institute for the Physics and Mathematics of the Universe (WPI), The University of Tokyo, Kashiwa 277-8583, Japan. ${ }^{9}$ Columbia University, 538 West 120th Street, New York, NY 10027, USA. ${ }^{10}$ Graduate School of Artificial Intelligence and Science, Rikkyo University, 3-34-1 Nishi Ikebukuro, Toshima, Tokyo 171-8501, Japan. ${ }^{11}$ Barnard College, 3009 Broadway, New York, NY 10027, USA. ${ }^{12}$ Department of Physics, The University of Tokyo, 7-3-1 Hongo, Bunkyo, Tokyo 113-0033, Japan. ${ }^{13}$ Research Center for the Early Universe, The University of Tokyo, 7-3-1 Hongo, Bunkyo, Tokyo 113-0033, Japan ${ }^{14}$ Massachusetts Institute of Technology, 77 Massachusetts Ave, Cambridge, MA 02139, USA. ${ }^{15}$ RIKEN Nishina Center, 2-1 Hirosawa, Wako, Saitama 351-0198, Japan. 